\section{La Révolution française}

Cahiers de l'Institut d'histoire de la Révolution française

$20 \mid 2021$

La Révolution en 3D - Textes, images, sons

(1787-2440)

\title{
Une fiction de la Révolution : Le projet de « La tenture Olympe de Gouges » (2009-?)
}

\section{Florence Lotterie}

\section{(2) OpenEdition \\ Journals}

Édition électronique

URL : https://journals.openedition.org//rf/4900

DOI : $10.4000 /$ Irf. 4900

ISSN : 2105-2557

Éditeur

IHMC - Institut d'histoire moderne et contemporaine (UMR 8066)

Référence électronique

Florence Lotterie, « Une fiction de la Révolution : Le projet de « La tenture Olympe de Gouges » (2009-

?) », La Révolution française [En ligne], 20 | 2021, mis en ligne le 25 juin 2021, consulté le 28 juin 2021.

URL : http://journals.openedition.org//rf/4900 ; DOI : https://doi.org/10.4000/Irf.4900

Ce document a été généré automatiquement le 28 juin 2021.

(c) La Révolution française 


\title{
Une fiction de la Révolution : Le projet de « La tenture Olympe de Gouges »(2009- ?)
}

\author{
Florence Lotterie
}

\section{NOTE DE L'ÉDITEUR}

Cet article est basé sur une communication à trois voix présentée lors du colloque « La Révolution en 3D - Textes, images, sons (1787-2440) » qui s'est tenu à l'université Paris 1 Panthéon-Sorbonne du 14 au 16 mars 2019, organisé par le Cespra et l'IHMC-IHRF. Vous pouvez retrouver cette communication sur la chaîne YouTube de l'IHMC à l'adresse : https://youtu.be/21p_I6mYDn8

\section{Introduction}

1 Jacques Fadat, né en 1950, est déjà le créateur de plus de trois cents tapisseries d'Aubusson. Il avait déjà réalisé et fait réaliser La Suite des Droits de l'homme en 1989. L'idée de cette nouvelle tenture lui est d'ailleurs venue du Bicentenaire, en voyant l'œuvre alors commandée par Paris à l'artiste coréen Nam Juke Paik, Olympe de Gouges dans la Fée électronique (qui se trouve dans les collections du Musée d'art moderne de la Ville de Paris). Il s'agissait d'un dispositif de téléviseurs organisés en «robotsréincarnations"; Gouges en faisait partie, en compagnie de Rousseau, Voltaire, Diderot, mais aussi Robespierre et Marat, avec lesquels elle ne se fût naturellement guère souciée de voisiner, voire de se trouver «interconnectée »: sous ce terme, le propos de l'œuvre était de manifester une "famille des robots de la Révolution ». Autant dire que Gouges, comme les autres, n'est alors qu'un nom propre qui fait mémoire et que, en ce sens l'art contemporain est aussi capable d'académiser que d'autres... Quoi qu'il en soit, Fadat s'est alors passionné pour Gouges et a lu ses principaux biographes, comme Olivier Blanc. Il lance le projet en 2009, alors que la 
Tapisserie d'Aubusson entre au patrimoine mondial de l'humanité. La tenture Olympe de Gouges est visible depuis son site, richement informé1.

2 Dans sa chronologie immédiate, on peut considérer ce projet comme impliqué dans la demande de patrimonialisation et de canonisation qui dure depuis au moins 2007 (la panthéonisation était une promesse électorale, on s'en souvient, de Ségolène Royal) jusqu'à 2018 (entrée au Panthéon de Simone Veil et de figures de la Résistance, choisies de préférence par Hollande en dépit d'une forte demande pour Gouges). Nous sommes devant un exemple, apparemment plutôt positif, de la réception paradoxale et à contretemps de Gouges, qui est passée d'un oubli saisissant ${ }^{2}$ (lequel concerna aussi la Déclaration de 1791, texte dont la diffusion fut plus que limitée dans son moment, alors qu'elle fut nettement plus significative aux États-Unis) à une sorte de "royauté iconique $^{3}$ " menaçant parfois de ne reposer sur aucune perception claire de l'œuvre, pour ne garder que l'icône. Encore est-elle, sur ce terrain, largement concurrencée par Théroigne. Il s'agit ici de présenter rapidement ce projet de tenture en deux temps. On rendra d'abord compte de la dimension positive de ce qui se présente comme un hommage : Gouges est célébrée à travers un objet qu'on peut présenter comme une contribution à l'histoire des conquêtes des femmes. On se penchera ensuite sur les ambiguïtés de cet hommage.

\section{Une histoire positive et conquérante des droits des femmes}

3 Fadat présente la tapisserie comme un moyen spécifique et particulièrement efficace de faire trace dans une monumentalité assumée. Il s'agit d'un enregistrement de la mémoire des sociétés et de leur fonds culturel, mais aussi de sa transmission, qui est en soi une fonction anthropologique fondamentale du tissage. Le point de rencontre entre l'objet de la tenture et son dispositif se situe au carrefour de dimensions explicites du projet: la mise en évidence de la qualité artistique du métier de lissier et la convergence des valeurs humanistes du compagnonnage ${ }^{4}$ avec la célébration d'une histoire positive des femmes.

4 Décrivons d'abord le dispositif. Les dix-sept articles de la Déclaration sont inscrits dans dix-sept panneaux distincts, où ils sont illustrés par le portrait d'une femme considérée pour son exemplarité dans une certaine histoire des femmes. De plus, chaque tapisserie est «marrainée » par une femme d'aujourd'hui dont l'action, l'œuvre, le métier, la notoriété, sont également considérés comme exemplaires d'un parcours lié à l'émancipation féminine. Par exemple, l'article premier de la Déclaration des droits de la femme est illustré dans son panneau par la figure de Joséphine Baker; et le panneau a pour marraine la danseuse étoile Marie-Claude Pietragalla. Le dispositif met ainsi en évidence une certaine histoire des femmes à partir de deux niveaux: celui de l'hommage (dans la tapisserie) et celui du patronage (dans le monde actuel) - ou plutôt faudrait-il dire « matronage », bien que cela ne soit pas la terminologie du projet.

5 Une chaîne se manifeste ainsi, où l'idée de la transmission historique emprunte une expression métaphorique à la technique même du tissage. Car Olympe de Gouges est ici la figure inspiratrice d'une sorte de "famille " féminine, à deux niveaux: par des héritières supposées (les femmes «tapissées») et par une instance qui vient symboliquement protéger et confirmer ce «fil», la «marraine» contemporaine 
associée à chaque tapisserie. C'est ainsi que se rend lisible une «trame », portée par un dispositif d'imagerie à trois entrées (le texte et son autrice, l'héritière, la " marraine »), voire à quatre, car le «fil » est aussi tissé à travers l'écran des femmes d'exception, vers ces «silences de l'Histoire ${ }^{5}$ » où s'inscrivent entre autres un «matrimoine » invisible ${ }^{6}$. Ainsi, cette histoire positive et ouverte reste-t-elle tout de même marquée par l'hypothèque spécifiquement féminine de la non-reconnaissance ou de l'oubli des plus nombreuses, inscrivant clairement l'entreprise dans un mouvement de réhabilitation et de réparation de cette « défiance des traces » dont parlait naguère Michelle Perrot.

Deux générations de femmes marquantes, ce serait peut-être un accident; mais à partir de trois, l'effet sériel est assuré, et ouvre sur une « histoire » qui suit son cours : les dixsept articles de la Déclaration seraient alors la circonscription apparente d'un texte qui, de lui-même, prouve son pouvoir de dissémination par le jeu de miroitement de ces femmes dans des strates temporelles et des sphères d'influence différentes. Entre la figure représentée et la "marraine ", il peut s'agir d'une continuité analogique : pour l'article III, par exemple, un «fil» européen se tisse avec Nicole Fontaine (décédée depuis). Mais ce peut être, plus directement, une sorte de filiation quasi littérale : Louise Weiss, choisie comme illustration pour la tapisserie de ce même article III, entretient une troublante ressemblance physique avec Nicole Fontaine... Ambiguïté, ou volonté de dire que «fille de » manifeste un héritage qui n'est pas un népotisme mais une solidarité nécessaire ${ }^{7}$ ? C'est en tout cas bien cela qui permet le « retour » à Gouges elle-même, dans l'hommage de ses filles et petites-filles, et c'est bien sûr ce qui fait que la série autorise aussi le "canon »: la chaine dit qu'une tradition de reconnaissance existe et peut s'ordonner en figures de savoir (Claudie Haigneré) et de pouvoir (Nicole Fontaine).

7 Le statut auctorial de Gouges est du même coup, comme c'est souvent le cas dans la mémoire commune, ici simplement confirmée, rapporté à l'identification « autrice de la Déclaration des droits des femmes ». Il serait sans doute juste de dire que c'est une des voies de sa réhabilitation dans le contexte de l'histoire du féminisme. Un livre de Léopold Lacour aujourd'hui oublié, sauf des spécialistes de la question ${ }^{8}$, Les origines $d u$ féminisme contemporain. Trois femmes de la Révolution. Olympe de Gouges, Théroigne de Méricourt, Rose Lacombe (Paris, Plon, 1900), illustre de manière exemplaire comment la disqualification de l'autrice littéraire $e^{9}$ peut " s'adosser à la reconnaissance de son apport politique; si l'autrice revient, c'est alors par le théâtre, comme celle qui y a défendu les droits des opprimés (les femmes et les noirs) par certaines pièces ».

8 Mais la symbolique de cette tenture confirme encore autrement la dimension généalogique conférée à la place de Gouges, si présente dans la construction politique et idéologique du « canon » féministe. En effet, elle y trouve un statut d'autrice comme autorité de la «toile " générale; celle-ci se présente comme une augmentation - auteur vient aussi de augeo - et une amplification des principes de la Déclaration, par les possibles réalisés dans l'histoire de la présence publique des femmes, qu'il s'agisse des femmes représentées ou des marraines. La dramatisation du statut fondateur de la Déclaration tient à ce que le prophétisme de Gouges (qu'elle a elle-même souvent revendiqué) s'applique d'ailleurs à la principale intéressée : le droit de "mourir en révolutionnaire ${ }^{10} »$. C'est, en effet, Gouges elle-même qui sert d'illustration au fameux article $\mathrm{X}$ : « Nul ne doit être inquiété pour ses opinions, même fondamentales, la femme a le droit de monter sur l'échafaud; elle doit avoir également celui de monter à la 
tribune, pourvu que ses manifestations ne troublent pas l'ordre public établi par la Loi. »

9 Ici, stratégiquement presque au centre de la série de tapisseries, le combat pour l'égalité des sexes retrouve sa modalité sacrificielle, paradoxale si l'on envisage la tenture comme une manifestation d'un temps positivement orienté de la conquête des droits, mais d'autant plus importante que l'un des enjeux de la mémoire de Gouges dans les ouvrages qui lui sont consacrés est, depuis son exécution, la décision de lui accorder ou (plus souvent) de lui retirer le courage de la fermeté révolutionnaire devant la mort. La « marraine » de cet article est Élisabeth Badinter, d'abord parce qu'on est ici au cœur de la question de l'égalité devant la loi et que Badinter autorise le "retour à Gouges » en tant que philosophe qui pense la question de l'égalité des sexes dans l'articulation avec celle des droits. Rappelons donc aussi, même s'il s'agit d'un point connu, que cette égalité, qui fonde la citoyenneté pleine et entière des femmes, n'est pensable que si elle intègre la nécessité, pour toute femme, de répondre de ses actes devant la loi et les tribunaux au même titre que les hommes, et pour encourir les mêmes risques pénaux (articles VII et IX). Comment ne pas saisir, ici, la curieuse résonance entre cette égalité devant la mort et le fait que le nom même de Badinter renvoie aussi à la mémoire politique de l'abolition de la peine capitale ? À cela près qu'il s'agit de Robert et que la résonance supposerait aussi de considérer Élisabeth comme « épouse de »...

\section{Un dispositif ambivalent}

Un tel dispositif attire l'attention sur la qualité de transmission d'une voix qui porte ce que l'on pourrait appeler une performativité historique : non seulement ce qui a été pensé et écrit se matérialise dans des trajectoires effectives et des œuvres au cours du temps long, mais ces trajectoires peuvent se subsumer sous l'exemplarité de la vie, des engagements et des sacrifices au nom du sexe d'Olympe de Gouges. Pourtant, ce modèle rencontre quelques discordances. Sur le site dédié à l'entreprise, les hommages voisinent avec quelques inexactitudes qui s'expliquent généralement par le souci même de situer le projet dans le cadre de la visibilisation d'une généalogie féminine. C'est ainsi qu'on peut lire à propos de Gouges que c'est la Déclaration «qui lui vaut d'être guillotinée ", notation du reste paradoxale si l'on entend insister sur le risque féministe de l'action politique d'Olympe de Gouges, topos historiographique qui appelle certaines nuances. Car c'est plutôt l'insistance à mettre en cause la Convention, et singulièrement Robespierre, autant qu'à produire des propositions politiques très imprudentes et insolentes dans leur contexte, qui a perdu Gouges. On pense ici à l'affiche des Trois urnes qui provoque son arrestation : en juillet 1793, en appeler à un scrutin national sur le choix de la forme du gouvernement (monarchie incluse) ne pouvait guère susciter autre chose qu'un retour de bâton répressif ${ }^{11}$. Ce qui la perd se fait donc par voie de placards, forme-discours en quête de visibilité publique avec laquelle la tapisserie aurait pu être l'occasion de marquer une certaine parenté, ou de suggérer certains échos ${ }^{12}$. Un tel mode de célébration, de toute façon, ne permet guère de faire affleurer la question de la nature même du "féminisme "supposément fondé par Olympe de Gouges en ses éventuelles apories politiques.

11 Du côté des historien-ne's, les choses ne sont du reste pas si claires. D'une part, on peut regretter que les figures - désormais si voyantes - de Gouges, Théroigne et autres Manon Roland ne soient précisément l'arbre qui cache la forêt de femmes tout aussi 
investies et bien plus nombreuses qu'on ne pourrait le croire ${ }^{13}$. À cet égard, l'analyse même de leurs prises de parole publique a été un objet historiographique longtemps négligé, et largement redevable à des travaux fondateurs d'historiennes ${ }^{14}$. Plus généralement, l'attention portée à Gouges et l'insistance, dans le contexte même de la demande de panthéonisation, sur son sacrifice comme femme, a pu heurter une tradition historiographique attentive à la complexité de l'investissement politique des femmes à la fin de l'Ancien Régime et dans le tournant révolutionnaire. Réagissant à un article de presse qui célébrait en Gouges une femme morte pour ses engagements féministes dans un contexte jacobino-républicain structurellement misogyne ${ }^{15}$, Florence Gauthier, soucieuse de pouvoir réfuter aussi dans cette thèse la doxa désormais vulgarisée des analyses de Joan Scott sur le caractère sexiste de l'universalisme républicain français ${ }^{16}$, avait indiqué, comme nous l'avons rappelé plus haut, que l'arrestation et l'exécution de Gouges relevait bien d'un motif politique, dans le contexte d'une lutte contre l'expression royaliste contre-révolutionnaire, motif qu'elle jugeait étranger à toute considération de sexe ; mais elle avait aussi inscrit les positions sociales et politiques de Gouges dans le cadre d'un girondinisme étranger aux droits des «subalternes »: des femmes du peuple, des pauvres et des esclaves. Féministe, Gouges? La question semblait plutôt ici celle de son accord avec la logique censitaire qui, en 1791, avait du même mouvement exclu les femmes et ceux qui ne pouvaient répondre du marc d'argent ${ }^{17}$.

12 Si l'on ne peut reprocher sans mauvaise foi à l'entreprise de cette Tenture de ne pas être à même de réfracter ces conflits interprétatifs, ce qui ne saurait être son objet, rien n'empêche de souligner que sa fonction par définition consensuelle fait contraste avec ce qui se passe dans la discussion historique. Au demeurant, jusqu'où avons-nous affaire à un geste féministe? La présentation de la tenture s'accompagne notamment d'entretiens filmés avec les "marraines", où l'on voit la complexité d'un travail mémoriel dont se donnent à entendre la diversité et les éventuelles dissonances : il ne s'agit pas d'une continuité lisse, si l'on ose dire, et cela aussi, une tapisserie le dit. La fille de Lucie Aubrac, par exemple, se félicite, dans une hésitation significative, de cette illustration d'un "mouvement féminin... féministe ", ajoutant d'ailleurs : « même si ça vient d'un homme». Elle exprime alors plutôt la caractéristique centripète de cet « entre femmes » et, à sa façon, une question que connaissent bien les historien'ne·s, dans le partage parfois critique entre " histoire des femmes » et " histoire du genre » s'impliquant dans les rapports de sexe ${ }^{18}$.

13 Mais on pourrait également poser la question de cette visibilité de femmes après tout blanches et privilégiées, et souvent elles-mêmes, comme Gouges, dans leur propre cercle professionnel, inscrites dans le cadre restreint de ce que Geneviève Fraisse appelle la «clause d'exceptionnalité ». Les travaux des historien.ne's mettent plus volontiers l'accent sur le fait que de telles figures sont l'arbre susceptible de cacher la forêt des anonymes et d'une pluralité des voix plus riche qu'on ne pourrait le penser : la question est alors celle de la place des révolutionnaires "ordinaires" et des subalternes dans l'historiographie ${ }^{19}$. Plus largement, le dispositif tend à un geste classique de célébration qui, tout en mettant en évidence des réussites singulières, ne permet pas vraiment de penser ces exemples dans leur articulation à une histoire vraiment collective des femmes, y compris dans leur diversité problématique, obligeant notamment à instruire la question sociale et coloniale ${ }^{20}$. Le projet de Jacques Fadat pourrait plutôt faire de lui un frère lointain de Léopold Lacour et autres représentants 
du «féminisme masculin» de la $\mathrm{III}^{\mathrm{e}}$ république étudié par Florence Rochefort et quelques autres, ambivalences incluses.

D'ailleurs, qui fabrique, ou est $v u$ fabriquer, sur les vidéos du site, la tenture qu'il a conçue ? Ce sont les lissiers et lissières de Maubusson, mais si on voit une photographie de lissière sur le site, c'est à «l'élite des lissiers » qu'on rend hommage et ce sont des hommes qui sont filmés en interview, les «maîtres lissiers". Là encore, il faut s'entendre. Jacques Fadat n'est aucunement responsable du fait que les hommes sont, dans ces métiers d'art très prestigieux, les chefs. C'est là une très vieille histoire des femmes et des métiers. En revanche, le projet, qui se réclame, dans l'Association, comme on l'a déjà noté, des "valeurs du compagnonnage", peut ici révéler, volontairement ou - sans doute plutôt - à son insu, les ratés du temps historique de la conquête des droits égaux. Dans l'une des vidéos de présentation sur le site, Catherine Allégret laisse ainsi échapper avec satisfaction, parlant de l'objet tapisserie : "On revient aux choses manuelles. »Ce qui peut aussi entrer en collision avec un autre imaginaire de la tapisserie - au mieux, les complexités du mythe de Philomèle, au pire, les ouvrages de dames.

Car l'ambiguïté tient aussi à la façon dont on peut inscrire le projet dans l'histoire de l'art textile. Pourquoi, sinon parce qu'il est de lui-même pour partie un produit hybride de cette histoire ${ }^{21}$, ne dit-il rien de son enjeu féministe ? N'a-t-elle pas vu, à partir du $\mathrm{xVIII}^{\mathrm{e}}$ siècle, les femmes se saisir stratégiquement de la dimension d'abord domestique de tels «ouvrages de dames» pour construire leur présence dans l'espace public de l'art? Le choix du grand format (voire de la monumentalité) n'a-t-il pas constitué un enjeu genré de cette conquête d'une présence ${ }^{22}$ ? Ces questions sont certes là, mais si elles flottent à la surface de notre perception devant un tel projet, force est de constater qu'elles y constituent d'étranges impensés, qui font notamment hésiter à lui accoler le terme de «femmage ${ }^{23}$ ».

Dans ce croisement du textuel et du sexuel, on voit en tout cas que l'histoire féministe des femmes et du genre reste ouverte sur ce qui manque encore (un peu ?) à sa place.

\section{Conclusion}

17 Tous ces éléments, finalement, renvoient à la construction même d'un dispositif qui, en faisant dialoguer plusieurs temporalités, sait aussi nous dire que les choses n'avancent pas en ligne droite. Dans La Citoyenne paradoxale (1998), qui commence avec Gouges, Joan Scott mettait en garde contre un récit qui serait trop "téléologique » de la conquête de l'égalité, d'autant qu'il faut se méfier des contextes discursifs, où "femme» et «féministe» ne veulent pas dire la même chose selon les époques. On peut avoir en tête cette mise en garde en contemplant la tapisserie dont George Sand constitue la figure : l'article VI de la Déclaration évoque en effet le droit de suffrage et d'accès aux emplois publics avec, en guise de symbole, une femme qui refusa, dans une lettre fameuse, d'assumer une candidature à la députation que des femmes révolutionnaires voulaient pour elle. Encore cette lettre elle-même illustre-t-elle une intelligence historique des temps (qui sont ou non venus) car Sand y dit qu'elle ne refuse pas le droit de vote aux femmes en soi, mais parce que ce droit ne pourrait s'exercer pleinement et lucidement que si les femmes pouvaient être maitresses de leur jugement et de leurs décisions, ce que le « contrat sexuel ${ }^{24}$ » du temps, appuyé sur la domination conjugale, ne permet pas encore. 
18 La tenture ne serait-elle pas un imaginaire du " pas encore » autant que du « déjà acquis »? Olympe de Gouges pourrait ainsi être une figure efficace du sens de l'histoire et de l'ouverture de ses possibles telles que les avaient pensées celui qu'on lui associe si souvent dans les origines «doctrinales » du féminisme, Condorcet, aussi bien qu'elle pourrait être un leurre. En outre, s'attachant à des figures d'illustres, le projet rejoint le style de canonisation propre à l'imaginaire (genré) des Lumières, celui du "grand homme ", dans lequel Gouges elle-même rêva (et revendiqua explicitement) de situer sa propre reconnaissance ${ }^{25}$. Un hommage, pas un femmage, donc.

\section{NOTES}

1. http://www.olympedegouges.fr/

2. Voir ici même la contribution d'Olivier Ritz.

3. L'expression, due à Jean-Claude Bonnet, désignait plutôt Voltaire et Rousseau («Les vies brèves de Diderot ", dans N. Lavialle et J.-B. Puech (dir.), L'Auteur comme ceuvre: l'auteur, ses masques, son personnage, sa légende, Presses universitaires d'Orléans, 2000, p. 51). Il est vrai que l'imagerie du "patriarche de Ferney» n'a aucune commune mesure avec les figurations d'Olympe de Gouges qui nous sont parvenues. Elles se sont toutefois multipliées, et il conviendrait sans doute d'associer à ce regain mémoriel le roman graphique de Catel et Boquet, Olympe de Gouges, Paris, Casterman, 2012.

4. Le projet est inscrit dans le Cercle de la Tapisserie d'Aubusson.

5. Michèle PERROT, Les Femmes ou les silences de l'Histoire, Paris, Flammarion, 1998.

6. http://www.olympedegouges.fr/hommages-marraines.html : « Notre présent est modelé par l'action de femmes exceptionnelles. Certaines d'entre elles sont les marraines de la "tenture Olympe de Gouges". Elles rendent hommage à d'autres femmes illustres dans les domaines où elles se sont distinguées. Elles célèbrent aussi à travers elles toutes les femmes inconnues qui, souvent en silence, élaborent des sociétés un peu plus harmonieuses. »

7. On notera, par ailleurs, que cette parenté s'exprime dans le dispositif: pour l'article VII, l'illustration est la Simone Signoret de Casque d'or (1952) et la marraine est sa propre fille, Catherine Allégret.

8. Voir notamment la riche étude de Florence ROCHEFORT, « Un féminisme radical au masculin à la Belle Époque: Le cas de Léopold Lacour ", dans Florence Rochefort et Éliane Viennot (dir.), L'Engagement des hommes pour l'égalité des sexes, Saint-Étienne, PUSE, 2013, p. 167-180.

9. Quoiqu'instruite par un intellectuel "féministe ", cette disqualification intervient en des termes qui n'ont rien à envier à la topique antiféministe habituelle, qui fait de Gouges une exaltée aux limites de la démence. Lacour demeure tout de même en-deçà de la littérature psychiatrique du moment 1900, où elle devient un cas d'école d'une supposée "paranoïa reformatoria » : voir Alfred GUILLoIs, Étude médico-psychologique sur Olympe de Gouges. Considérations générales sur la mentalité des femmes pendant la Révolution française, Paris, Rey, 1904. Guillois fait naturellement l'objet de citations indignées chez les principaux biographes contemporains de Gouges (Olivier Blanc, Benoîte Groult).

10. Nous empruntons ici son beau titre au colloque organisé à Rouen par Michel Biard, Philippe Bourdin, Laurent Brassart, Jean-Numa Ducange, Jean-Yves Frétigné, Hervé Leuwers, Côme Simien 
et Cyril Triolaire, Mourir en révolutionnaire (XVIII ${ }^{e}-X^{e}$ siècle), Université de Rouen, 25-28 novembre 2019.

11. Ainsi que l'a encore rappelé récemment Olivier Blanc, Olympe de Gouges a paru pleinement persuadée que son initiative ne pouvait tomber sous le coup de la loi du 29 mars 1793 (et non du 28 comme mentionné) qui punit de mort les écrits tendant au rétablissement de la royauté (Olivier BLANC, « Olympe de Gouges. Un nouveau modèle d'engagement citoyen », dans M. Poirson (dir.), Amazones de la Révolution. Des femmes dans la tourmente de 1789, Gourcuff Gradenigo, Montreuil, 2016, p. 76). Elle est toutefois condamnée au titre de l'article I ${ }^{\text {er }}$ de cette loi.

12. La symbolique du support est tout de même incomparablement plus en phase avec l'engagement d'olympe de Gouges que l'offre, rapidement retirée devant l'indignation des internautes, de la boutique en ligne de l'Assemblée nationale, qui avait proposé en 2015 un tablier de cuisine dont l'imprimé était précisément le texte de la Déclaration de 1791. Parmi les réactions, ce tweet irrésistible du journal Causette : «La femme a le droit de monter sur l'échafaud; elle doit avoir également celui de monter la mayonnaise. »

13. Appuyé sur de nombreux travaux classiques, ce constat est encore celui que le cinéaste Pierre Schoeller a pu mettre en avant pour justifier son intérêt pour les femmes révolutionnaires dans son dernier film, Un peuple et son roi (2017).

14. Voir les travaux classiques de Dominique Godineau et de Martine Lapied, ainsi que Christine FAURÉ, «Doléances, déclarations et pétitions, trois formes de la parole publique des femmes sous la Révolution », AHRF, n 344, 2006, p. 5-25.

15. Myriam PERFETTI, «Olympe de Gouges, une femme contre la Terreur », Marianne, 17-26 août 2013, p. 76-79.

16. Joan sсотt, La Citoyenne paradoxale. Les féministes françaises et les droits de l'homme, Paris, Albin Michel, « Bibliothèque Histoire », 1998.

17. Florence GAUTHIER, « Olympe de Gouges, histoire ou mystification? », https://www.pouruneconstituante.fr/spip.php?article1597

18. Pour des mises au point récentes sur ces enjeux disciplinaires, voir Dominique GODINEAU et alii, «Femmes, genre, révolution», AHRF, n³58, 2009, p. 143-166; Guillaume MAZEAU et Clyde PLUMAUZILLE, « Penser avec le genre : Trouble dans la citoyenneté révolutionnaire ", La Révolution française [En ligne], 9|2015, mis en ligne le 16 novembre 2015, consulté le 09 décembre 2019. URL : http://journals.openedition.org/lrf/1458; DOI : 10.4000/lrf.1458.

19. À titre d'exemple, le livre récent de Martine Lapied peut apparaitre comme une manière de décaler le regard sur l'identité « provençale » des femmes investies en politique, même si on a pu lui reprocher de pas "descendre " suffisamment dans les couches populaires (Martine LAPIED, L'Engagement politique des femmes dans le sud-est de la France de l'Ancien Régime à la Révolution, Université d'Aix-Marseille Éditions, 2019). Ce souci des voix non entendues est également au cœur de l'entreprise de Clyde PLumauziLle, Prostitution et révolution. Les femmes publiques dans la cité républicaine (1789-1804), Seyssel, Champ Vallon, coll. « La Chose publique », 2016.

20. L'exception du dispositif, de ce point de vue, est certes voyante puisqu'elle se trouve à son ouverture (L'article premier illustré par J. Baker). Mais n'est-elle pas voyante comme exception?

21. Au sens où il manifeste en lui-même que la noblesse de l'art reste dans une large mesure un marqueur masculin. Pour le dire autrement : le monde de la tapisserie reste en partie tributaire de cette logique que Christine Planté a analysée, à propos de la littérature, comme « le genre des genres » littéraires (Voir notamment Christine PLANTÉ, La petite sœur de Balzac. Essai sur la femme auteur, ré-éd. Presses universitaires de Lyon, 2015 [1989]). C'est bien la tapisserie comme pratique et support sexués qui est paradoxale ici, où l'on est au croisement de l'art et de l'artisanat.

22. Chaque tapisserie fait $2,30 \mathrm{~m} \times 2,30 \mathrm{~m}$. On sait que le petit format fut longtemps l'apanage (si l'on peut dire) des femmes en art et il a été en partie lié à la nature des travaux «d'aiguille »; et que le passage au format monumental dans l'art textile est aussi un mode de manifestation 
féministe chez un certain nombre d'artistes : à cet égard, s'il y a une œuvre en particulier à confronter à la Tenture, c'est peut-être le fameux (et controversé) Dinner Party de Judy Chicago. Sur ces questions liées à l'art textile, voir en particulier Rozsika PARKER, The Subversive Stitch. Embroidery and the Making of the Feminine, London, The Women's Press, 1984.

23. Historiquement, le «femmage " («Female Art of Collage», à propos des œuvres de Miriam Shapiro) s'inscrit comme geste d'artiste femme en lien de filiation et/ou de reconnaissance à d'autres femmes dans le cadre d'un art «textile» précisément défini par ses origines, ses matériaux et ses procédures hybrides, mixtes, entre «noblesse " esthétique et trivialité du quotidien.

24. Voir Carole PATEMAN, Le Contrat sexuel, Paris, La Découverte, 2010 [1988].

25. Sur cet imaginaire, voir Jean-Claude BONNET, Naissance du Panthéon. Essai sur le culte des grands hommes, Paris, Fayard, 1998 ; sur son importance dans le discours gougien, voir notamment Jessica goodman (dir.), Commemorating Mirabeau: Mirabeau aux Champs Elysées and other texts, Cambridge, MHRA, 2017.

\section{RÉSUMÉS}

L'article analyse la construction d'un objet à la confluence de l'art, de l'artisanat, des pratiques mémorielles et du geste commémoratif : le projet d'une vaste «tenture », conçue par l'artiste Jacques Fadat, formée de plusieurs panneaux visant à célébrer, à travers le texte de sa Déclaration des droits de la femme, la figure d'olympe de Gouges. À travers un dispositif d'associations de cette figure à des femmes caractérisées comme des emblèmes de l'émancipation, la tenture lui confère une fonction tutélaire dans une histoire longue, contribuant ainsi à une forme d'historiographie des apports de la Révolution à l'affirmation progressive des femmes, non sans qu'apparaissent alors des tensions problématiques.

This article analyses the construction of an object at the intersection of art, craft, and memorial practices: the project of a vast wall hanging, conceived by the artist Jacques Fadat, composed of several panels aiming to celebrate, through the text of her Declaration of the Rights of Women, the figure of Olympe de Gouges. Through a system of associations between this figure and women characterised as emblems of emancipation, the hanging confers on her a tutelary function in a long history, thus taking part in a form of historiography about the contribution of the French Revolution to the progressive affirmation of women, but not without problematic tensions arising.

Olympe de Gouges, History of Representations, Women and the French Revolution, Celebration

\section{INDEX}

Mots-clés : Olympe de Gouges, Histoire des représentations, Femmes et Révolution française, Commémoration 
AUTEUR

FLORENCE LOTTERIE

CERILAC - EA 4410

Université de Paris 